\title{
Complete Controllability of Linear Fractional Differential Systems with Singularity
}

\author{
Qun Huang, Qiuyue Wu, Xian-Feng Zhou, Yanhua Wen, and Jiang Wei
}

School of Mathematical Sciences, Anhui University, Hefei 230039, China

Correspondence should be addressed to Xian-Feng Zhou; zhouxf@mail.ustc.edu.cn

Received 28 February 2015; Revised 8 May 2015; Accepted 10 May 2015

Academic Editor: Sotiris K. Ntouyas

Copyright (C) 2015 Qun Huang et al. This is an open access article distributed under the Creative Commons Attribution License, which permits unrestricted use, distribution, and reproduction in any medium, provided the original work is properly cited.

\begin{abstract}
This paper is concerned with the controllability of a class of linear fractional differential systems with singularity. The method which is used to deal with the fast subsystem $N \cdot{ }^{c} D_{0, t}^{\alpha} x_{2}(t)=x_{2}(t)+B_{2} u(t)$ and $y_{2}(t)=C_{2} x_{2}(t)$ is an improvement of the known ones. Based on the movement orbit of the state equation, we obtain several controllability criteria which are sufficient and necessary.
\end{abstract}

\section{Introduction and Preliminaries}

Singular systems are also commonly called descriptor systems, generalized state-space systems, differential-algebraic systems, or semistate systems whose behaviors are described by differential equations (or difference equations) and algebraic equations. In the past few decades, singular systems have attracted much attention for their extensive applications in robotics [1], power systems [2], networks, economic systems [3,4], highly interconnected large-scale systems [5], and so on. Many fundamental notions and conclusions based on regular systems have been extended to singular systems. For detail, see the monographs $[6,7]$.

The concept of controllability plays an important role in the analysis and design of control systems. Recently, the controllability of fractional differential systems has been gaining much attention. For example, by applying Schauder's fixed point theorems, the authors of the paper [8] obtained a set of sufficient conditions for the controllability of nonlinear fractional differential systems. Using Sadovskii's fixed point theorem and Krasnoselskii's fixed point theorem, respectively, and properties of characteristic solution operators, the authors of the paper [9] established the complete controllability criteria for fractional evolution systems. Without involving the compactness of characteristic solution operators, the authors of the paper [10] considered the controllability of nonlinear dynamical systems with time varying multiple delays and distributed delays, respectively. In the paper [11], the controllability of fractional impulsive neutral integrodifferential systems was investigated. By using Krasnoselskii's fixed point theorem and the properties of resolvent operators, sufficient conditions for the controllability were established. By the representation of the state solution and construction of suitable control inputs, the authors established the controllability criteria for a class of linear neutral fractional timeinvariant differential systems. These criteria are sufficient and necessary [12]. In 2013, the paper [13] was concerned with the controllability of fractional functional evolution equations of Sobolev type in Banach space. With the help of two characteristic solution operators and their properties, the authors obtained the controllability criteria corresponding to two admissible control sets via the well-known Schauder's fixed point theorem. In 2014, the paper [14] dealt with the controllability of Sobolev type fractional evolutions and some controllability criteria were derived.

However, as far as we know, little attention has been paid to singular fractional differential systems. Motivated by this fact, this paper is devoted to the controllability for the singular fractional differential system

$$
\begin{aligned}
E \cdot{ }^{c} D_{0, t}^{\alpha} x(t) & =A x(t)+B u(t), \quad t \geq 0, \\
y(t) & =C x(t),
\end{aligned}
$$

where ${ }^{c} D_{0, t}^{\alpha}$ is Caputo's derivative of order $\alpha$ with the lower limit $0,0<\alpha<1, E, A \in \mathbb{R}^{n \times n}$ are $n \times n$ constant matrices, the matrix couple $(E, A)$ is regular which will be defined later, 
$B \in \mathbb{R}^{n \times m}$ and $C \in \mathbb{R}^{r \times n}$ are the known constant matrices, $\operatorname{rank} E \triangleq q<n, x \in \mathbb{R}^{n}$ is the state variable, $u \in \mathbb{R}^{m}$ is the control input, and $y \in \mathbb{R}^{r}$ is the output.

Under the assumption that the matrix couple $\left(\begin{array}{ll}E & A\end{array}\right)$ is regular, there exist two nonsingular matrices $Q$ and $P$ satisfying

$$
\begin{aligned}
Q E P & =\operatorname{diag}\left(\begin{array}{ll}
I_{n_{1}} & N
\end{array}\right), \\
Q A P & =\operatorname{diag}\left(\begin{array}{ll}
A_{1} & I_{n_{2}}
\end{array}\right), \\
C P & =\left(\begin{array}{ll}
C_{1} & C_{2}
\end{array}\right), \\
Q B & =\left(\begin{array}{c}
B_{1} \\
B_{2}
\end{array}\right),
\end{aligned}
$$

where $N \in \mathbb{R}^{n_{2} \times n_{2}}$ is nilpotent whose index is $h$ (namely, $h$ is the smallest integer such that $\left.N^{h}=0\right), A_{1} \in \mathbb{R}^{n_{1} \times n_{1}}, B_{1} \in$ $\mathbb{R}^{n_{1} \times m}, B_{2} \in \mathbb{R}^{n_{2} \times m}, C_{1} \in \mathbb{R}^{r \times n_{1}}$, and $C_{2} \in \mathbb{R}^{r \times n_{2}}$. Then system (1) can be written as

$$
\begin{aligned}
{ }^{c} D_{0, t}^{\alpha} x_{1}(t) & =A_{1} x_{1}(t)+B_{1} u(t), \\
y_{1}(t) & =C_{1} x_{1}(t), \\
N \cdot{ }^{c} D_{0, t}^{\alpha} x_{2}(t) & =x_{2}(t)+B_{2} u(t), \\
y_{2}(t) & =C_{2} x_{2}(t), \\
y & =y_{1}+y_{2},
\end{aligned}
$$

where $P^{-1} x=\left(\begin{array}{l}x_{1} \\ x_{2}\end{array}\right), x_{1} \in \mathbb{R}^{n_{1}}, x_{2} \in \mathbb{R}^{n_{2}}$, and $n_{1}+n_{2}=n$. Subsystems (3)-(4) and (5)-(6) are called the slow subsystem and the fast subsystem, respectively.

Note that subsystem (3)-(4) is a normal fractional differential linear system, whose controllability has been discussed in [15].

Hence, in order to investigate the controllability of system (1), it is sufficient to investigate the controllability of subsystem (5)-(6), which is our main task in this paper.

Before giving our main results, we first recall some definitions and lemmas. For more details, please refer to $[6,16,17]$.

Definition 1 (see [6]). For any given two matrices $E$ and $A \in$ $\mathbb{R}^{n \times n}$, the matrix couple $(E A)$ is called regular if there exists a constant scalar $a \in \mathbb{C}$ such that $|a E+A| \neq 0$ or the polynomial $|s E-A| \not \equiv 0$.

Lemma 2 (see [15]). The slow subsystem (3)-(4) of system (1) is controllable if rank $\left(\begin{array}{lllll}B_{1} & A_{1} B_{1} & \cdots & A_{1}^{n_{1}-1} B_{1}\end{array}\right)=n_{1}$.

Definition 3 (see [16]). Given an interval $[a, b]$ of $\mathbb{R}$, the fractional order integral of a function $f \in L^{1}[a, b]$ of order $\alpha \in \mathbb{R}$ is defined by

$$
\begin{aligned}
I_{a, t}^{\alpha} f(t)=\frac{1}{\Gamma(\alpha)} \int_{a}^{t}(t-s)^{\alpha-1} f(s) d s, & \\
& t \in[a, b], \alpha>0,
\end{aligned}
$$

where $\Gamma$ is the Gamma function.
Definition 4 (see [16]). Suppose that a function $f$ is defined on the interval $[a, b]$ and $f^{(n)}(t) \in L^{1}[a, b]$. Caputo's fractional derivative of order $\alpha$ with lower limit $a$ for $f$ is defined as

$$
\begin{aligned}
{ }^{c} D_{a, t}^{\alpha} f(t) & =\frac{1}{\Gamma(n-\alpha)} \int_{a}^{t}(t-s)^{n-\alpha-1} f^{(n)}(s) d s \\
& =I_{a, t}^{n-\alpha}\left(f^{(n)}(t)\right), \quad t \in[a, b],
\end{aligned}
$$

where $0<n-1<\alpha \leq n$.

Particularly, when $0<\alpha<1$, it holds that

$$
\begin{aligned}
{ }^{c} D_{a, t}^{\alpha} f(t) & =\frac{1}{\Gamma(1-\alpha)} \int_{a}^{t}(t-s)^{-\alpha} f^{\prime}(s) d s \\
& =I_{a, t}^{1-\alpha} f^{\prime}(t), \quad t \in[a, b] .
\end{aligned}
$$

The Laplace transform of Caputo's fractional derivative ${ }^{c} D_{0, t}^{\alpha} x(t)$ is

$$
\begin{aligned}
L\left[{ }^{c} D_{0, t}^{\alpha} x(t) ; s\right] & =\int_{0}^{+\infty} e^{-s t}\left({ }^{c} D_{0, t}^{\alpha} x(t)\right) d t \\
& =s^{\alpha} \bar{x}(s)-\sum_{k=0}^{n-1} s^{\alpha-k-1} x^{(k)}(0), \\
& n-1<\alpha \leq n,
\end{aligned}
$$

where $\bar{x}(s)$ is the Laplace transform of $x(t)$.

Particularly, for $0<\alpha<1$, it holds that

$$
\int_{0}^{+\infty} e^{-s t}\left({ }^{c} D_{0, t}^{\alpha} x(t)\right) d t=s^{\alpha} \bar{x}(s)-s^{\alpha-1} x(0) .
$$

In addition, the Laplace transforms of the $k$ th derivative of $u(t)$ and the $k$ th derivative of the Dirac function $\delta(t)$ are

$$
\begin{aligned}
& L\left[u^{(k)}(t) ; s\right]=s^{k} \bar{u}(s)-\sum_{i=0}^{k-1} s^{i} u^{(k-i-1)}(0), \\
& L\left[\delta^{(k)}(t) ; s\right]=s^{k}, \quad k \in \mathbb{N}^{+}=\{1,2, \ldots\}
\end{aligned}
$$

and if $\alpha \in \mathbb{R} \backslash \mathbb{N}^{+}$, then the Laplace transform of $t^{-\alpha-1}$ is

$$
L\left[t^{-\alpha-1} ; s\right]=\Gamma(-\alpha) s^{\alpha}, \quad \alpha \in \mathbb{R} \backslash \mathbb{N}^{+}, \operatorname{Re}(s)>0 .
$$

Lemma 5 (see [17]). Let $0<\operatorname{Re}(\alpha) \leq 1$. If $x(t) \in A C[a, b]=$ $\left\{x \mid x(t):[a, b] \rightarrow \mathbb{R}^{n}\right.$ is absolutely continuous $\}$ or $x(t) \in$ $C[a, b]=\left\{x \mid x(t):[a, b] \rightarrow \mathbb{R}^{n}\right.$ is continuous $\}$, then

$$
I_{a, t}^{\alpha}\left({ }^{c} D_{a, t}^{\alpha} x(t)\right)=x(t)-x(a) .
$$

Throughout this paper, " $|M|$ " denotes the norm of the matrix " $M$ ", " $M^{T}$ " denotes the transpose of the matrix " $M$ ", $\mathbb{C}$ denotes the complex plane, and " $\Leftrightarrow$ " denotes equivalence.

\section{State Response}

In this section, the state response of system (5)-(6) is given by the following theorem. 
Theorem 6. Assume that $h$ is the nilpotent index of the matrix N. Suppose further that the function $u(t)$ is $h$ times continuously differentiable and $u^{(i)}(0)=0, i=1,2, \ldots$.. Then the distribution solution of the state equation (5) has the following form:

$$
x_{2}(t)=-\sum_{k=1}^{h-1} N^{k} g_{k}(t) x_{2}(0)-\sum_{k=0}^{h-1} N^{k} B_{2} f_{k}(t),
$$

where

$$
\begin{aligned}
f_{0}(t) & =u(t), \\
g_{k}(t) & =L^{-1}\left[s^{k \alpha-1} ; t\right], \\
f_{k}(t) & =L^{-1}\left[s^{k \alpha} \bar{u}(s) ; t\right], \\
k & =1,2, \ldots, h-1 .
\end{aligned}
$$

Here $\left.x_{2}(t)\right|_{t=0}=x_{2}(0)$ and $\bar{u}(s)$ is the Laplace transform of $u(t)$. Namely, $\bar{u}(s)=L[u(t) ; s] . L^{-1}[\bar{u}(s) ; t]$ denotes the inverse Laplace transform of $\bar{u}(s)$.

Proof. An application of the Laplace transform on both sides of (5) yields

$$
N \cdot\left(s^{\alpha} \bar{x}_{2}(s)-s^{\alpha-1} x_{2}(0)\right)=\bar{x}_{2}(s)+B_{2} \bar{u}(s),
$$

where $\bar{x}_{2}(s)$ and $\bar{u}(s)$ are the Laplace transform of $x_{2}(t)$ and $u(t)$, respectively. That is

$$
\begin{aligned}
\bar{x}_{2}(s)= & \left(N s^{\alpha}-I\right)^{-1} N s^{\alpha-1} x_{2}(0) \\
& +\left(N s^{\alpha}-I\right)^{-1} B_{2} \bar{u}(s) .
\end{aligned}
$$

Since $N \in \mathbb{R}^{n_{2} \times n_{2}}$ is nilpotent whose index is $h$ and

$$
\begin{aligned}
& \left(s^{\alpha} N-I\right)^{-1}=-\left(I+s^{\alpha} N+s^{2 \alpha} N^{2}+\cdots\right. \\
& \left.\quad+s^{(h-2) \alpha} N^{h-2}+s^{(h-1) \alpha} N^{h-1}\right)
\end{aligned}
$$

we get

$$
\begin{gathered}
\bar{x}_{2}(s)=\left(N s^{\alpha}-I\right)^{-1} N s^{\alpha-1} x_{2}(0)+\left(N s^{\alpha}-I\right)^{-1} \\
\cdot B_{2} \bar{u}(s)=-\left(s^{\alpha-1} N+s^{2 \alpha-1} N^{2}+s^{3 \alpha-1} N^{3}+\cdots\right. \\
\left.+s^{(h-1) \alpha-1} N^{h-1}+s^{h \alpha-1} N^{h}\right) x_{2}(0)-\left(I+s^{\alpha} N\right. \\
\left.+s^{2 \alpha} N^{2}+\cdots+s^{(h-2) \alpha} N^{h-2}+s^{(h-1) \alpha} N^{h-1}\right) B_{2} \bar{u}(s) .
\end{gathered}
$$

Now, we consider the following two cases.

Case (i). $\nu \in \mathbb{N}^{+}=\{1,2, \ldots\}$ and $u^{i}(0)=0, i=1,2, \ldots, \nu$. By formula (13), we have

$$
\begin{aligned}
L^{-1}\left[s^{\nu} ; t\right] & =\delta^{(v)}(t), \\
L^{-1}\left[s^{\nu} \bar{u}(s) ; t\right] & =u^{(v)}(t) .
\end{aligned}
$$

Case (ii). $v \in \mathbb{R} \backslash \mathbb{N}^{+}$. By formula (14), we have

$$
L^{-1}\left[s^{\nu} ; t\right]=\frac{1}{\Gamma(-\nu)} t^{-\nu-1} .
$$

Applying the inverse Laplace transform and Convolution Theorem yields

$$
L^{-1}\left[s^{\nu} \bar{u}(s) ; t\right]=\frac{1}{\Gamma(-\nu)} \int_{0}^{t}(t-\tau)^{-\nu-1} u(\tau) d \tau .
$$

Finally, in order to obtain the desired results, it is sufficient to apply the inverse Laplace transform on both sides of (21) together with formulas (22)-(24). This completes the proof.

Remark 7. Obviously, the output response $y_{2}(t)$ is given by

$$
\begin{aligned}
y_{2}(t) & =C_{2} x_{2}(t) \\
& =-\sum_{k=1}^{h-1} C_{2} N^{k} g_{k}(t) x_{2}(0)-\sum_{k=0}^{h-1} C_{2} N^{k} B_{2} f_{k}(t) .
\end{aligned}
$$

\section{Controllability}

In this section, we will establish some controllability criteria for subsystem (5)-(6) and system (1). We begin with the concept of the controllability of subsystem (5)-(6).

Definition 8. System (5)-(6) is called controllable if, for any $t_{1}>0, x_{2}(0) \in \mathbb{R}^{n_{2}}$, and $w \in \mathbb{R}^{n_{2}}$, there exists a control input $u(t) \in C_{p}^{h-1}, x_{2}\left(t_{1}\right)=w$. Here $C_{p}^{h-1}$ denotes the set of the $h-1$ times piecewise continuously differentiable functions.

The following lemma is well-known.

Lemma 9. Consider two given constant matrices: $A \in \mathbb{R}^{n \times n}$ and $B \in \mathbb{R}^{n \times m}$. The following statements are equivalent:

(1) $\operatorname{rank}(s I-A B)=n, \forall s \in \mathbb{C}$, where $\mathbb{C}$ represents the complex plane

(2) $\operatorname{rank}\left(\lambda_{i} I-A B\right)=n$, where $\lambda_{i}$ are the eigenvalues of matrix $A$;

(3) $\operatorname{rank}\left(\begin{array}{llll}B & A B & \cdots & A^{n-1} B\end{array}\right)=n$.

The following two theorems and a corollary are our main results of this paper.

Theorem 10. The slow subsystem (3)-(4) of system (1) is controllable if and only if one of the following holds:

(a) $\operatorname{rank}\left(B_{1} A_{1} B_{1} \cdots A_{1}^{n_{1}-1} B_{1}\right)=n_{1}$;

(b) $\operatorname{rank}\left(s I-A_{1} \quad B_{1}\right)=n_{1}$;

(c) $\operatorname{rank}(s E-A B)=n, \forall s \in \mathbb{C}$, where $\mathbb{C}$ represents the complex plane.

Proof. By Lemma 2, the slow subsystem (3)-(4) is controllable if and only if (a) holds.

$$
\text { (a) } \Leftrightarrow(\text { b). By Lemma 9, (a) and (b) are equivalent. }
$$


(b) $\Leftrightarrow(\mathrm{c})$. In fact,

$$
\begin{aligned}
& \operatorname{rank}(s E-A, B)=\operatorname{rank}(s Q E P-Q A P Q B) \\
& =\operatorname{rank}\left(\begin{array}{ccc}
s I-A_{1} & O & B_{1} \\
O & s N-I & B_{2}
\end{array}\right) \\
& =\operatorname{rank}\left(\begin{array}{ccc}
s I-A_{1} & B_{1} & O \\
O & O & s N-I
\end{array}\right) \\
& =n_{2}+\operatorname{rank}\left(\begin{array}{ll}
s I-A_{1} & B_{1}
\end{array}\right) \text {, }
\end{aligned}
$$

where the matrices $Q$ and $P$ satisfy (2). Obviously, (b) and (c) are equivalent. This completes the proof.

Theorem 11. The fast subsystem (5)-(6) of system (1) is controllable if and only if one of the following holds:
(a) $\operatorname{rank}\left(\begin{array}{llll}B_{2} & N B_{2} & \cdots & N^{h-1} B_{2}\end{array}\right)=n_{2}$;
(b) $\operatorname{rank}\left(\begin{array}{ll}N & B_{2}\end{array}\right)=n_{2}$;
(c) $\operatorname{rank}\left(\begin{array}{ll}E & B\end{array}\right)=n$.

Proof. We first prove that subsystem (5)-(6) is controllable if and only if (a) holds. According to the state response of the fast subsystem (5)-(6), we have

$$
\begin{aligned}
x_{2}(t) & +\sum_{k=1}^{h-1} N^{k} g_{k}(t) x_{2}(0) \\
& =-\sum_{k=0}^{h-1} N^{k} B_{2} f_{k}(t) \\
& =\left(\begin{array}{llll}
B_{2} & N B_{2} & \cdots & N^{h-1} B_{2}
\end{array}\right)\left(\begin{array}{c}
f_{0}(t) \\
f_{1}(t) \\
\vdots \\
f_{h-1}(t)
\end{array}\right),
\end{aligned}
$$

where $f_{k}(t)$ and $g_{k}(t)$ are satisfying (17). For any $t_{1}>0$, $x_{2}(0) \in \mathbb{R}^{n_{2}}$ and $w_{2} \in \mathbb{R}^{n_{2}}$, the sufficient and necessary condition to have a control input $u(t) \in C_{p}^{h-1}$ such that $x_{2}\left(t_{1}\right)=w_{2}$ is $\operatorname{rank}\left(B_{2} N B_{2} \cdots N^{h-1} B_{2}\right)=n_{2}$. Thus subsystem (5)-(6) is controllable if and only if (a) holds.

Now we prove the equivalence between (a) and (b). Since $N$ is nilpotent, $\Delta(N)=\left\{\lambda_{i}\left|\lambda_{i} \in \mathbb{C},\right| \lambda_{i} I-N \mid=\right.$ $0\}=\{0\}$. By the equivalence of (2) and (3) in Lemma 9, $\operatorname{rank}\left(B_{2} \quad N B_{2} \cdots N^{h-1} B_{2}\right)=n_{2}$ holds if and only if $\operatorname{rank}\left(\begin{array}{ll}-N & B_{2}\end{array}\right)=\operatorname{rank}\left(\begin{array}{cc}N & B_{2}\end{array}\right)=n_{2}$.

Finally, we prove the equivalence between (b) and (c). Note the fact that

$$
\begin{aligned}
\operatorname{rank}\left(\begin{array}{ll}
E & B
\end{array}\right) & =\operatorname{rank}\left(\begin{array}{ll}
Q E P & Q B
\end{array}\right) \\
& =\operatorname{rank}\left(\begin{array}{ccc}
I_{n_{1}} & O & B_{1} \\
O & N & B_{2}
\end{array}\right) \\
& =n_{1}+\operatorname{rank}\left(\begin{array}{ll}
N & B_{2}
\end{array}\right)
\end{aligned}
$$

where the matrices $Q$ and $P$ satisfy (2). Obviously, (b) and (c) are equivalent. This completes the proof.

By Theorems 10 and 11, the following corollary yields.

Corollary 12. System (1) is controllable if and only if $\operatorname{rank}(s E-A B)=n$ and $\operatorname{rank}\left(\begin{array}{ll}E & B\end{array}\right)=n, \forall s \in \mathbb{C}$.

\section{Illustrative Examples}

Example 1. Consider system (1). Assume that $\alpha=1 / 2$, and

$$
\begin{aligned}
E & =\left(\begin{array}{cccc}
3 & 0 & 0 & 0 \\
0 & 5 & 0 & 0 \\
0 & 0 & -2 & 0 \\
0 & 0 & 0 & 0
\end{array}\right), \\
A & =\left(\begin{array}{cccc}
0 & 0 & 0 & 1 \\
0 & 0 & 1 & 0 \\
-1 & 1 & 0 & 0 \\
1 & 0 & 0 & 3
\end{array}\right), \\
B & =\left(\begin{array}{c}
0 \\
0 \\
0 \\
-1
\end{array}\right) .
\end{aligned}
$$

By computation, we have

$$
\begin{aligned}
\operatorname{rank}(s E-A \quad B) & =\operatorname{rank}\left(\begin{array}{ccccc}
3 s & 0 & 0 & -1 & 0 \\
0 & 5 s & -1 & 0 & 0 \\
1 & -1 & -2 s & 0 & 0 \\
-1 & 0 & 0 & -3 & -1
\end{array}\right) \\
& =\operatorname{rank}\left(\begin{array}{ccccc}
0 & 0 & 0 & -1 & 0 \\
0 & 0 & -1 & 0 & 0 \\
1 & 0 & 0 & 0 & 0 \\
0 & 0 & 0 & 0 & -1
\end{array}\right)=4, \\
\operatorname{rank}\left(\begin{array}{ccccc}
E & B
\end{array}\right) & =\operatorname{rank}\left(\begin{array}{ccccc}
3 & 0 & 0 & 0 & 0 \\
0 & 5 & 0 & 0 & 0 \\
0 & 0 & -2 & 0 & 0 \\
0 & 0 & 0 & 0 & -1
\end{array}\right)=4 .
\end{aligned}
$$


Example 2. Consider system (1). Assume that $\alpha=1 / 2$, and

$$
\begin{aligned}
& E=\left(\begin{array}{lll}
0 & 1 & 0 \\
1 & 0 & 1 \\
0 & 0 & 0
\end{array}\right), \\
& A=\left(\begin{array}{lll}
1 & 0 & 0 \\
0 & 1 & 0 \\
0 & 0 & 1
\end{array}\right), \\
& B=\left(\begin{array}{l}
0 \\
1 \\
0
\end{array}\right), \\
& C=\left(\begin{array}{lll}
0 & 1 & 0
\end{array}\right) .
\end{aligned}
$$

By computation, we have

$$
\begin{aligned}
\operatorname{rank}(s E-A \quad B) & =\operatorname{rank}\left(\begin{array}{cccc}
-1 & s & 0 & 0 \\
s & -1 & s & 1 \\
0 & 0 & -1 & 0
\end{array}\right) \\
& =\operatorname{rank}\left(\begin{array}{cccc}
1 & 0 & 0 & 0 \\
0 & 0 & 0 & 1 \\
0 & 0 & 1 & 0
\end{array}\right)=3, \\
\operatorname{rank}\left(\begin{array}{llll}
E & B
\end{array}\right) & =\operatorname{rank}\left(\begin{array}{cccc}
0 & 1 & 0 & 0 \\
1 & 0 & 1 & 1 \\
0 & 0 & 0 & 0
\end{array}\right)=2<3 .
\end{aligned}
$$

By Corollary 12, system (1) is not controllable.

\section{Conflict of Interests}

The authors declare that there is no conflict of interests regarding the publication of this paper.

\section{Acknowledgments}

This paper is supported by Natural Science Foundation of China (11471015 and 11371027), Natural Science Foundation of Anhui Province (1508085MA01), Natural Science Foundation for Colleges and Universities of Anhui Province (KJ2012A032), Research Fund for Doctoral Program of Higher Education of China (20123401120001 and 20133401120013), and The Program of Academic Innovation Research for Postgraduate of Anhui University (yfc100013).

\section{References}

[1] J. K. Mills and A. A. Goldenberg, "Force and position control of manipulators during constrained motion tasks," IEEE Transactions on Robotics \& Automation, vol. 5, no. 1, pp. 30-46, 1989.

[2] R. W. Newcomb and B. Dziurla, "Some circuits and systems applications of semistate theory," Circuits, Systems, and Signal Processing, vol. 8, no. 3, pp. 235-260, 1989.
[3] D. G. Luenberger, "Dynamic equations in descriptor form," IEEE Transactions on Automatic Control, vol. 22, no. 3, pp. 312321, 1977.

[4] D. G. Luenberger and A. Arbel, "Singular dynamic Leontief systems," Econometrica, vol. 45, no. 4, pp. 991-995, 1977.

[5] Y. Q. Liu and Z. H. Guan, Stabilization and Control of Measure Large-Scale Systems with Impulses, South China University of Technology Press, Guangzhou, China, 1996.

[6] L. Y. Dai, Singular Control Systems, Springer, New York, NY, USA, 1989.

[7] S. Y. Xu and J. Lam, Robust Control and Filtering of Singular Systems, Springer, Berlin, Germany, 2006.

[8] K. Balachandran, J. Y. Park, and J. J. Trujillo, "Controllability of nonlinear fractional dynamical systems," Nonlinear Analysis. Theory, Methods \& Applications, vol. 75, no. 4, pp. 1919-1926, 2012.

[9] J. R. Wang and Y. Zhou, "Complete controllability of fractional evolution systems," Communications in Nonlinear Science and Numerical Simulation, vol. 17, no. 11, pp. 4346-4355, 2012.

[10] K. Balachandran, Y. Zhou, and J. Kokila, "Relative controllability of fractional dynamical systems with delays in control," Communications in Nonlinear Science and Numerical Simulation, vol. 17, no. 9, pp. 3508-3520, 2012.

[11] Z. Tai and S. Lun, "On controllability of fractional impulsive neutral infinite delay evolution integrodifferential systems in Banach spaces," Applied Mathematics Letters, vol. 25, no. 2, pp. 104-110, 2012.

[12] X.-F. Zhou, J. Wei, and L.-G. Hu, "Controllability of a fractional linear time-invariant neutral dynamical system," Applied Mathematics Letters, vol. 26, no. 4, pp. 418-424, 2013.

[13] M. Fečkan, J. Wang, and Y. Zhou, "Controllability of fractional functional evolution equations of Sobolev type via characteristic solution operators," Journal of Optimization Theory and Applications, vol. 156, no. 1, pp. 79-95, 2013.

[14] J. R. Wang, M. Fečkan, and Y. Zhou, "Controllability of Sobolev type fractional evolution systems," Dynamics of Partial Differential Equations, vol. 11, no. 1, pp. 71-87, 2014.

[15] D. Matignon and B. d'André-Novel, "Observer-based controllers for fractional differential systems," in Proceedings of the 36th IEEE Conference on Decision \& Control, pp. 4967-4972, IEEE, San Diego, Calif, USA, December 1997.

[16] Y.Zhou, Basic Theory of Fractional Differential Equations, World Scientific, Singapore, 2014.

[17] A. A. Kilbas, H. M. Srivastava, and J. J. Trujillo, Theory and Applications of Fractional Differential Equations, vol. 204 of North-Holland Mathematics Studies, Elsevier Science B.V., Amsterdam, The Netherlands, 2006. 


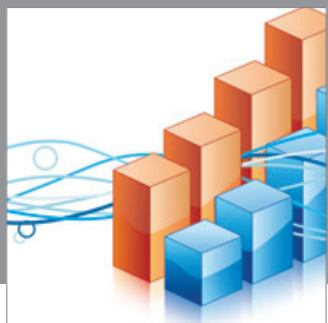

Advances in

Operations Research

mansans

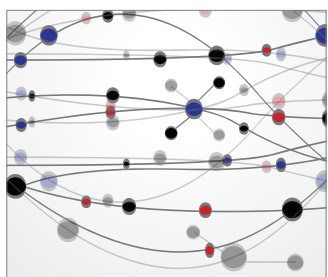

The Scientific World Journal
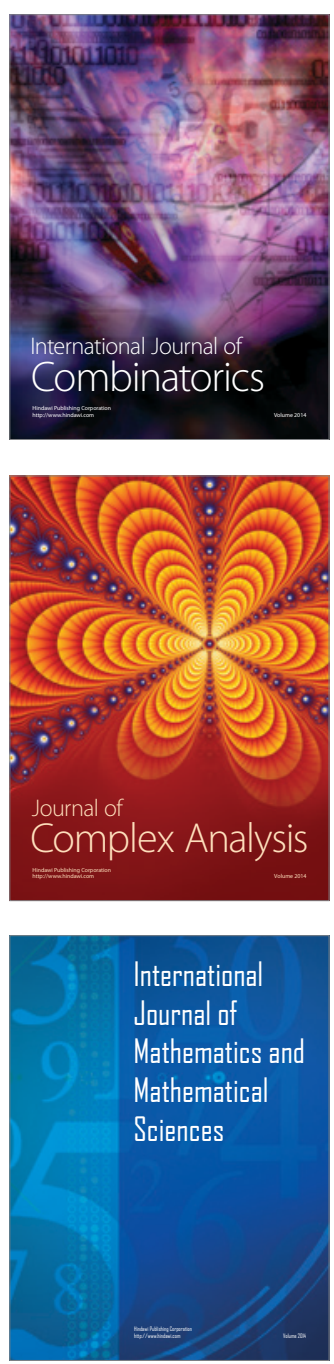
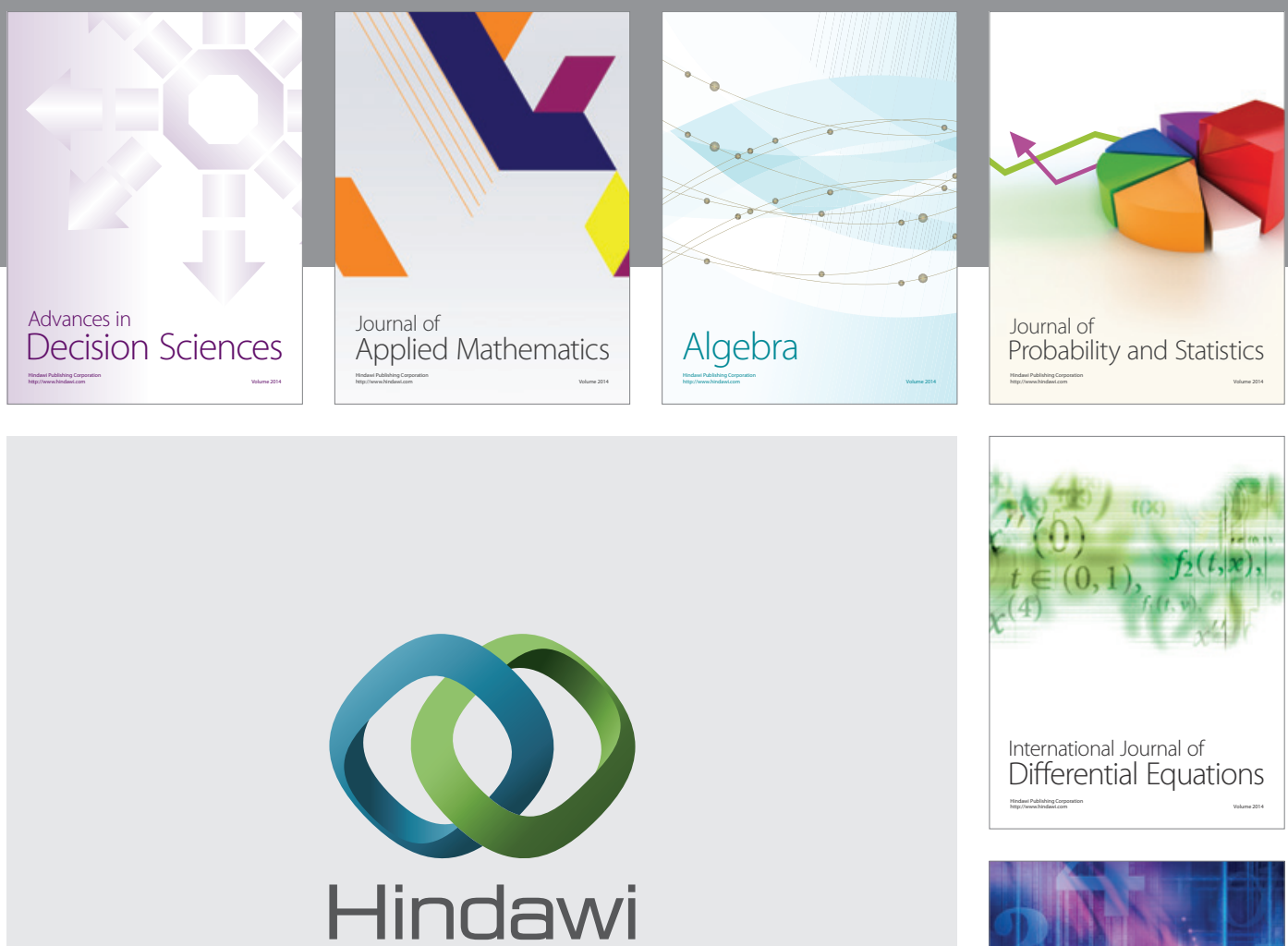

Submit your manuscripts at http://www.hindawi.com
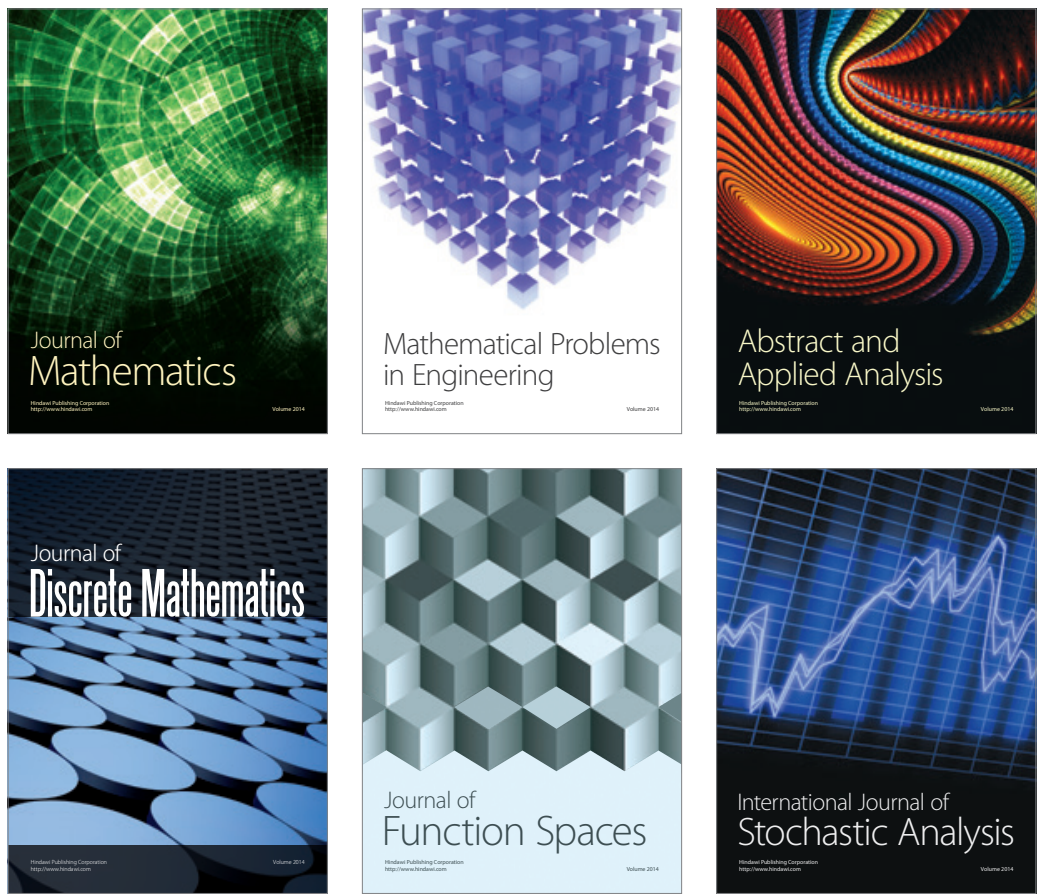

Journal of

Function Spaces

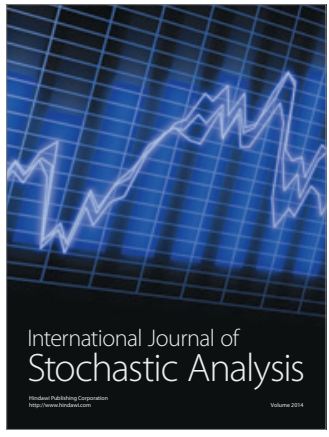

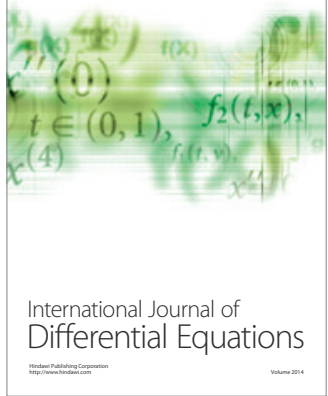
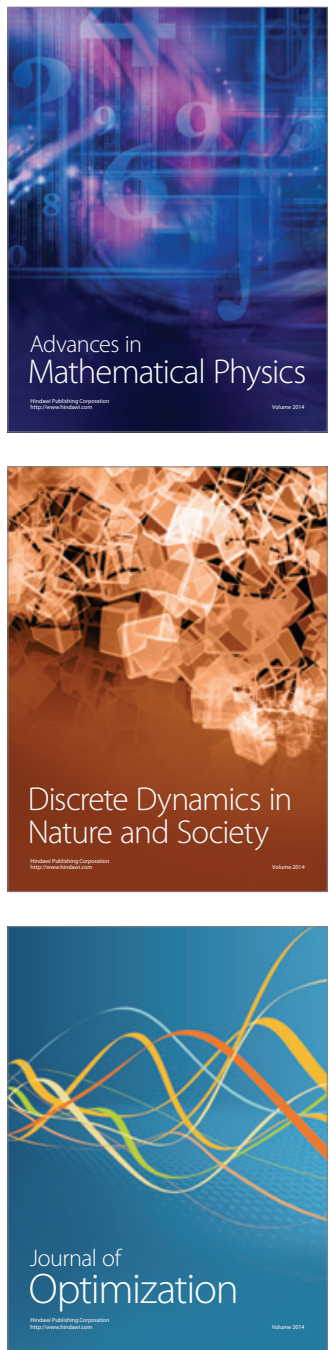